\title{
Anomalías vasculares más frecuentes en pacientes pediátricos. Parte 2: Malformaciones vasculares
}

\author{
Vascular anomalies in childhood. Part 2: Vascular \\ malformations
}

Blanca Lucina Campos-Cabrera, ${ }^{1}$ Edna Morán-Villaseñor, ${ }^{1}$ María Teresa García-Romero, ${ }^{1}$ Carola Durán-McKinster ${ }^{2}$

\section{Resumen}

Las anomalías vasculares (AV) comprenden un grupo heterogéneo de alteraciones en los vasos sanguíneos y linfáticos. La Sociedad Internacional para el Estudio de las Anomalías Vasculares (ISSVA) las clasifica en dos grupos: tumores y malformaciones vasculares. Las malformaciones vasculares son entidades secundarias a errores congénitos durante la morfogénesis vascular. De acuerdo con el vaso malformado se dividen en: capilares, linfáticas, venosas, arteriovenosas, fístulas arteriovenosas, malformaciones combinadas, de grandes vasos y asociadas a otras anomalías.

Las malformaciones capilares afectan piel y mucosas, se presentan como manchas de aspecto vascular, con mayor frecuencia en cabeza y cuello, cuyo color varía desde un tono rosado a un eritemato-violáceo.

Las malformaciones linfáticas se manifiestan como un incremento de volumen subcutáneo, bien delimitado, de consistencia blanda, sin cambios en la coloración de la piel subyacente. Las malformaciones venosas se presentan como un aumento de volumen mal delimitado, de consistencia suave y compresible, la piel de la superficie presenta una coloración azul-violácea y si bien son congénitas, pueden no ser evidentes hasta la pubertad o posterior a un traumatismo. Finalmente, las malformaciones arteriovenosas que son las menos frecuentes, se presentan como un incremento de volumen subcutáneo, mal delimitado, de consistencia firme con un thrill o pulso a la palpación. La importancia de este segundo artículo de AV radica en poder categorizar de una manera inicial, si se trata de un tumor o una malformación vascular, con el fin de ofrecer un abordaje y manejo interdisciplinario y dirigido.

PALABRAS CLAVE: Vasos linfáticos; tumores vasculares; malformaciones vasculares; hemangioma congénito; hemangioma piógeno; hemangioendotelioma; hemangioendotelioma kaposiforme; síndrome de Kasabach-Merritt.

\section{Abstract}

Vascular anomalies are a heterogeneous group of disorders that affect blood and lymphatic vessels. The International Society for the Study of Vascular Anomalies (ISSVA), classifies them in two groups: vascular tumors and vascular malformations.

Vascular malformations are entities secondary to congenital errors during vascular morphogenesis. They are divided according to the malformed vessel into: capillary, lymphatic, venous, arteriovenous, arteriovenous fistulas, combined malformations and malformations associated with other anomalies.

Capillary malformations affect skin and mucous membranes, and present as stains of vascular appearance, most often on the head and neck. Color varies from pink to erythematous-violaceous. Lymphatic malformations present with an increase in subcutaneous volume, well-defined, of soft consistency, without changes in the color of the skin. Venous malformations present as a poorly defined volume increase, with a soft and compressible consistency, the skin of the surface presents a blue-violet color and although they are congenital, they may not be evident until puberty or after
${ }^{1}$ Consulta privada, dermatóloga pediatra.

${ }^{2}$ Adscrita al servicio de Dermatología.

Instituto Nacional de Pediatría, Ciudad de México.

Recibido: 5 de agosto 2019

Aceptado: 12 de diciembre 2019

Correspondencia

Carola Durán McKinster

caroladmc53@gmail.com

Este artículo debe citarse como Campos-Cabrera BL, Morán-Villaseñor E, García-Romero MT, Durán-McKinster C. Anomalías vasculares más frecuentes en pacientes pediátricos. Parte 2: Malformaciones vasculares. Acta Pediátr Méx 2020;41(2):85-98. 
Finally, arteriovenous malformations, which are the least frequent, appear as poorly defined increase in subcutaneous volume, of firm consistency with a thrill or pulse on palpation. The importance of this second VA article lies on the ability to categorize whether it is a vascular tumor or a vascular malformation and offer a proper approach and treatment from the beginning.

KEYWORDS: Vascular anomalies; Vascular tumors; Vascular malformations.

\section{INTRODUCCIÓN}

Las anomalías vasculares comprenden un amplio grupo de alteraciones en los vasos sanguíneos y linfáticos. De acuerdo con la clasificación de la Sociedad Internacional para el estudio de las Anomalías Vasculares, ISSVA, por sus siglas en inglés (International Society for the Study of Vascular Anomalies), las anomalías vasculares se dividen en dos grandes grupos: tumores y malformaciones vasculares. ${ }^{1-4}$

Los tumores vasculares se caracterizan por presentar proliferación e hiperplasia de células endoteliales. En contraste, las malformaciones vasculares son secundarias a errores congénitos en la morfogénesis vascular, con un componente relativamente estático o quiescente. ${ }^{1}$

En este artículo (segunda parte de dos) se describen las malformaciones vasculares más frecuentes en pacientes pediátricos, incluyendo su fisiopatogenia, manifestaciones clínicas, diagnóstico y tratamiento, con el objetivo de promover el uso de la clasificación y terminología adecuada para una referencia y tratamiento oportuno por parte del pediatra o médico de primer contacto.

\section{GENERALIDADES DE LAS MALFORMACIONES VASCULARES}

Las malformaciones vasculares cutáneas son un grupo heterogéneo de trastornos localizados o diseminados que suceden durante el desarrollo de la morfogénesis vascular. Gran parte son esporádicas aunque algunas son hereditarias y tienden a manifestarse como lesiones multifocales. ${ }^{5}$ Su frecuencia es de $0.5-1.5 \%$ de la población general; las más frecuentes son las malformaciones capilares y venosas. No se ha reportado alguna predilección de género. ${ }^{2,6}$

En la patogenia de las malformaciones vasculares está involucrado tanto el proceso de vasculogénesis (formación de vasos sanguíneos) como el de angiogénesis (formación de nuevos vasos a partir de una vasculatura preexistente). ${ }^{7}$ Durante ambos procesos se han identificado diversos factores angiogénicos alterados: en el receptor del factor de crecimiento endotelial vascular-1 (VEGF-1) hay defectos que evitan la unión con su ligando correspondiente (VEGF), lo que condiciona un vaso de paredes delgadas. ${ }^{7,8}$ También se han identificado defectos en la angiopoyetina y en el factor de crecimiento transformante beta (TGF- $\beta$ ) que favorecen la proliferación de células endoteliales y la formación de vasos tortuosos. ${ }^{8,9}$

Las malformaciones vasculares se dividen, de acuerdo con el tamaño de la malformación y asociaciones, en cuatro grupos: simples, combinadas, de grandes vasos y asociadas con otras anomalías. ${ }^{1,4}$

En el Cuadro 1 se muestra la actualización más reciente de la clasificación de la ISSVA, modificada en la Reunión Bianual de 2018. Solo se incluyen las más frecuentes en pacientes pediátricos. La versión completa de esta clasificación se encuentra en www.issva.org. ${ }^{4}$ 
Cuadro 1. Clasificación de las anomalías vasculares ${ }^{4}$

\begin{tabular}{|c|c|}
\hline Tumores & Malformaciones \\
\hline Benignos & Simples \\
\hline Hemangioma infantil & Capilares \\
\hline Hemangioma congénito & Linfáticas \\
\hline $\begin{array}{l}\text { Rápidamente } \\
\text { involutivo }\end{array}$ & Venosas \\
\hline $\begin{array}{l}\text { Parcialmente } \\
\text { involutivo }\end{array}$ & Arteriovenosas \\
\hline No involutivo & Fístula arteriovenosa \\
\hline $\begin{array}{l}\text { Hemangioma capilar } \\
\text { lobulado (granuloma piógeno) }\end{array}$ & Combinadas \\
\hline Angioma en penacho* & $\begin{array}{l}\text { Dos o más malforma- } \\
\text { ciones vasculares }\end{array}$ \\
\hline $\begin{array}{l}\text { Localmente agresivos o } \\
\text { limítrofes }\end{array}$ & De grandes vasos \\
\hline $\begin{array}{l}\text { Hemangioendotelioma } \\
\text { kaposiforme* }\end{array}$ & $\begin{array}{l}\text { Asociadas con otras } \\
\text { anomalías }\end{array}$ \\
\hline Sarcoma de Kaposi & $\begin{array}{l}\text { Síndrome de } \\
\text { Klippel-Trenaunay }\end{array}$ \\
\hline $\begin{array}{l}\text { Angioendotelioma papilar } \\
\text { intralinfático (tumor de Dabska) }\end{array}$ & $\begin{array}{l}\text { Síndrome de } \\
\text { Parkes-Weber }\end{array}$ \\
\hline Malignos & $\begin{array}{l}\text { Síndrome de } \\
\text { Servelle-Martorell }\end{array}$ \\
\hline Angiosarcoma & $\begin{array}{l}\text { Síndrome de } \\
\text { Sturge-Weber }\end{array}$ \\
\hline \multirow[t]{8}{*}{$\begin{array}{l}\text { Hemangioendotelioma } \\
\text { epitelioide }\end{array}$} & $\begin{array}{l}\text { Malformación capilar } \\
\text { en extremidad asociada } \\
\text { a hipertrofia congénita } \\
\text { no progresiva }\end{array}$ \\
\hline & Síndrome de Maffucci \\
\hline & $\begin{array}{l}\text { Malformación } \\
\text { capilar-macrocefalia }\end{array}$ \\
\hline & $\begin{array}{l}\text { Malformación } \\
\text { capilar-microcefalia }\end{array}$ \\
\hline & Síndrome CLOVES \\
\hline & Síndrome de Proteus \\
\hline & $\begin{array}{l}\text { Síndrome de } \\
\text { Bannayan-Riley } \\
\text { Ruvalcaba }\end{array}$ \\
\hline & Síndrome CLAPO \\
\hline
\end{tabular}

Adaptado de ISSVA Classification for Vascular Anomalies, actualizada en el 2018 y disponible en issva.org/classification. *Algunos consideran a estas 2 entidades parte de un mismo espectro, más que dos entidades distintas.

\section{MALFORMACIONES VASCULARES SIMPLES}

Las malformaciones vasculares simples solo están compuestas por un tipo de vaso (capilar, linfático o vena), con excepción de las malformaciones arteriovenosas, las cuales están compuestas por arterias, venas y capilares; sin embargo, se incluyen en esta categoría. De igual forma las fístulas arteriovenosas se consideran dentro de este grupo. ${ }^{1,4}$

\section{Malformaciones capilares}

\section{Epidemiología}

Las malformaciones capilares son las malformaciones vasculares más frecuentes, afectan la piel y las mucosas; son de flujo lento y hemodinámicamente inactivas. ${ }^{2,6}$ La mancha salmón, o nevo simple, aparece en 20 a $80 \%$ de los recién nacidos $^{10}$ y representa un motivo frecuente de consulta. No tienen predominio de género. ${ }^{4,11}$

\section{Etiología}

Las malformaciones capilares suelen ser esporádicas, aunque hay casos familiares. ${ }^{4,6}$ Se han relacionado con mutaciones en el cromosoma $5 q$ que codifica para el gen RASA y con mutaciones somáticas activadoras en el gen GNAQ localizado en el cromosoma 9q21.11,12

\section{Manifestaciones clínicas}

Son manchas de aspecto vascular; su color varía de rosado a eritemato-violáceo, de límites bien definidos o de aspecto reticulado y de formas irregulares o geométricas. La topografía más frecuente es en la cabeza y el cuello y pueden ser malformaciones únicas o múltiples. ${ }^{4}$ Están presentes desde el nacimiento y se mantienen durante toda la vida. Con la edad, algunas pueden adquirir una coloración más violácea, 


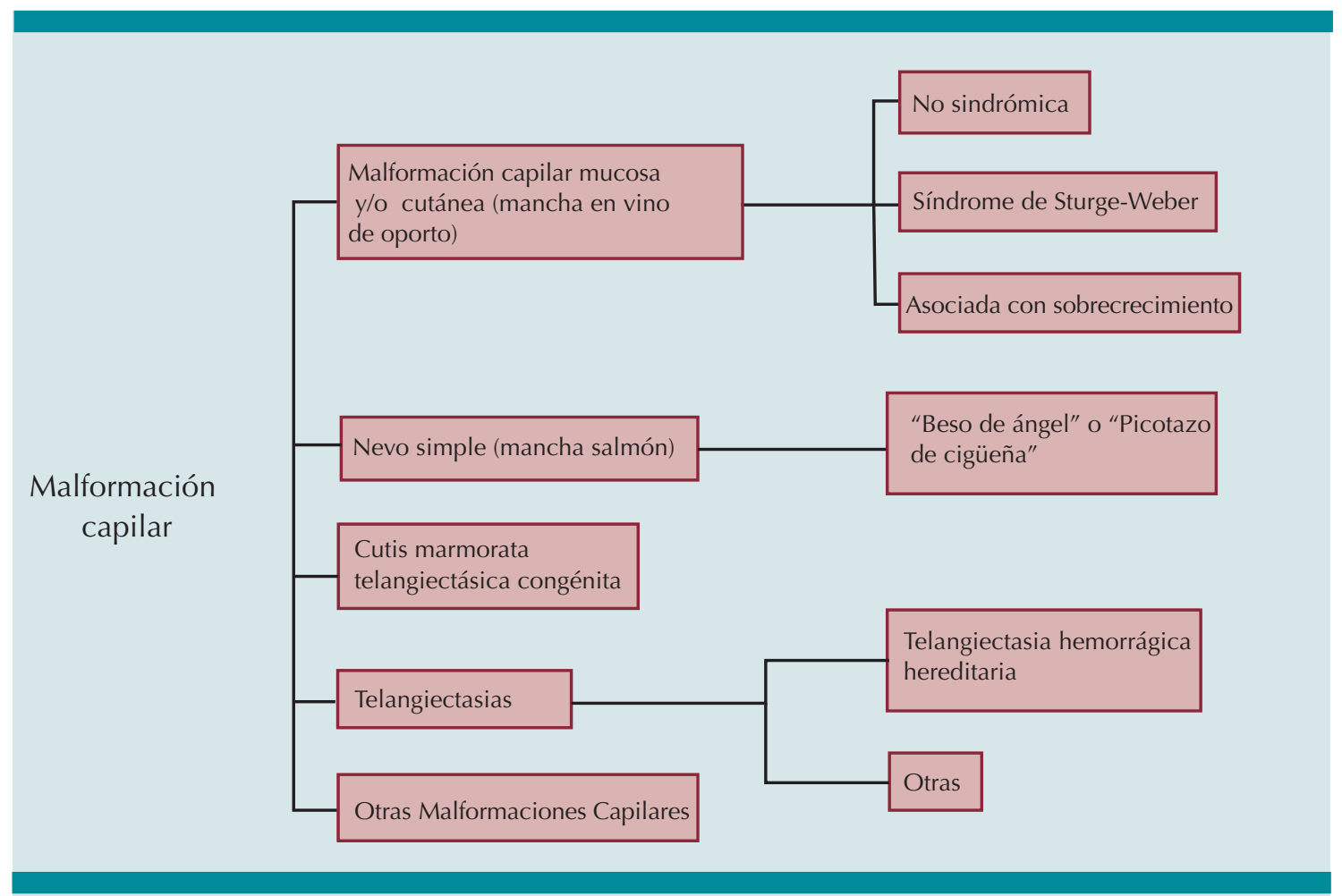

Cuadro 2. Clasificación de las malformaciones capilares (ISSVA 2018). ${ }^{4}$

mientras que otras pueden palidecer. Suelen tener un crecimiento proporcional al del paciente. $^{12}$

De acuerdo con sus características clínico-evolutivas y pronósticas, la ISSVA propone clasificar a las malformaciones capilares en cinco categorías. ${ }^{4}$ Cuadro 2

1. Mancha en vino de Oporto.

2. Mancha salmón o nevo simple.

3. Cutis marmorata telangiectásica congénita.

4. Telangiectasias.

5. Otras malformaciones capilares.

\section{Mancha en vino de Oporto o nevo flámeo}

Es una malformación capilar cutánea y/o mucosa que se presenta desde el nacimiento, pero a diferencia de la mancha salmón o nevo simple, no desaparece con el tiempo. Es la segunda malformación capilar más común, después del nevo simple; se observa en $0.3 \%$ de los recién nacidos. $^{2,11}$ Se manifiesta desde el nacimiento con una mancha de color rosado o rojiza que puede presentar varios patrones: bien definida, geográfica, reticulada y difusa o pequeña y múltiple. Por lo general tiene distribución segmentaria, unilateral y se localiza con mayor frecuencia en la cabeza y el cuello. El $60 \%$ de los pacientes presenta hipertrofia de tejidos blandos 
siendo más notoria en la tercera o cuarta década de la vida. En algunos pacientes el color se torna más oscuro con la edad y pueden formarse nódulos sobre la malformación capilar secundarios a ectasia vascular. Puede afectar los labios, la encía y el paladar, y con el tiempo desarrollar hipertrofia gingival. ${ }^{11,12}$

Alrededor de $45-80 \%$ de los niños con mancha en vino de Oporto segmentaria, que afecta la frente, tienen riesgo de síndrome de SturgeWeber: un trastorno neurocutáneo caracterizado por: malformación capilar (Figura 1), glaucoma y angiomatosis leptomeníngea. ${ }^{13,14}$ El $75-90 \%$ de los pacientes con síndrome de Sturge-Weber tienen crisis convulsivas en los primeros dos años de vida. Otras manifestaciones neurológicas incluyen: hemiparesia lentamente progresiva, migraña, retraso en el desarrollo y accidentes cerebrovasculares. La malformación leptomeníngea capilar-venosa predomina en forma ipsilateral a la mancha en vino de Oporto, pero también puede presentarse de forma bilateral. El glaucoma también tiende a ser unilateral e ipsilateral a la malformación capilar. ${ }^{15}$

De acuerdo con las características clínicas y radiológicas se han descrito tres tipos de síndrome de Sturge-Weber: tipo I (clásico) caracterizado por una malformación capilar en la cara y angiomatosis leptomeníngea con o sin glaucoma; el tipo II, que es el más frecuente, en el que hay una malformación capilar en la cara, sin afectación leptomeníngea, con o sin glaucoma y el tipo III en donde solo se encuentra angiomatosis leptomeníngea, siendo la presentación menos común. ${ }^{15,16}$

\section{Mancha salmón o nevo simple}

Predomina en la línea media de la cabeza, sobre todo en la glabela, frente, párpados o nuca; pueden tener una distribución en " $\mathrm{V}$ " y son de límites imprecisos (Figura 2). Se hacen más evidentes con el Ilanto o cambios de temperatura y suelen volverse más claras y desaparecer con

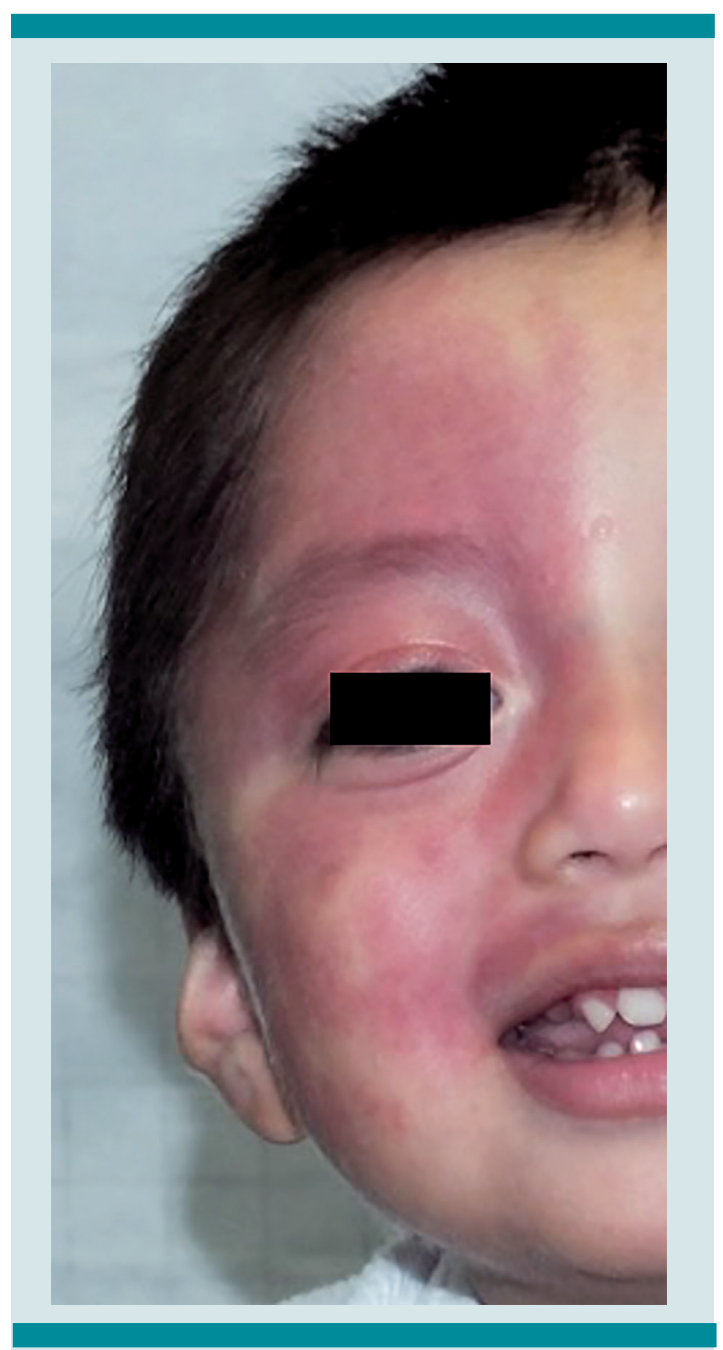

Figura 1. Síndrome de Sturge-Weber.

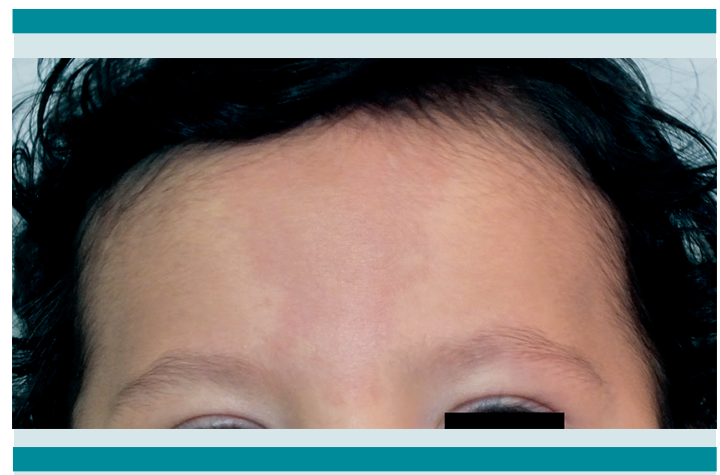

Figura 2. Mancha salmón o nevo simple en la frente. 
el tiempo, generalmente antes de los 5 años, excepto la malformación capilar en la nuca. . $^{411,12}$ Son conocidas coloquialmente como "beso de ángel" si se localizan en la frente o "picotazo de cigüeña" si está en la nuca. ${ }^{12,17,18}$ Cuando las malformaciones capilares se localizan en la región lumbosacra se denominan "manchas en mariposa" y cuando se sitúan a un lado de la línea media pueden asociarse con disrafismo espinal; por lo que es necesario la realización de estudios de imagen. ${ }^{19}$

\section{Cutis marmorata telangiectásica congénita}

En estos casos la malformación capilar es de color azul-violáceo, con un patrón reticulado acompañado de áreas de telangiectasias y piel atrófica. Pueden localizarse con una distribución segmentaria o generalizada. Lo más común es que afecte una extremidad. El $50 \%$ de los pacientes puede tener otras anomalías, sobre todo de crecimiento: hipo o hipertrofia. También se han descrito alteraciones craneoencefálicas, neurológicas, retinianas, cutáneas y óseas. Por lo general, disminuyen con el paso de los años, aunque pueden persistir toda la vida. ${ }^{12,20}$

\section{Telangiectasias}

Las telangiectasias se presentan como máculas rojas puntiformes, lesiones lineales o estrelladas o pápulas rojizas que desaparecen a la presión. La etiología es variada y deben diagnosticarse oportunamente, en particular la telangiectasia hemorrágica hereditaria (Rendu-Osler-Weber). ${ }^{21}$

En el grupo de las telangiectasias se encuentran:

- El nevo araña, una mancha rojiza con el centro más oscuro y telangiectasias radiales con predominio en la cara y las manos que representan solo una condición estética. ${ }^{6,21}$
- La telangiectasia hereditaria benigna se hereda en forma autosómica dominante con lesiones que predominan en la cara y el dorso de las manos. Se presenta en la edad pediátrica y no se asocia a alteraciones hemorrágicas. ${ }^{21}$

- La telangiectasia nevoide unilateral se manifiesta en forma de telangiectasias puntiformes segmentarias en la etapa prepuberal. ${ }^{5,21}$

- La telangiectasia esencial generalizada, la cual es muy rara, inicia en las extremidades y progresivamente se extiende a todo el cuerpo siendo asintomática. ${ }^{6,21}$

Si bien las malformaciones capilares se presentan generalmente de forma aislada, en forma no sindrómica, también se pueden presentar asociadas a otras anomalías como se mencionará más adelante. ${ }^{2}$

\section{Diagnóstico}

El diagnóstico de las malformaciones capilares es clínico. Los estudios de imagen están indicados en pacientes con sospecha de síndrome de Sturge-Weber en quienes se recomienda la resonancia magnética con gadolinio entre los 3 y 6 meses de edad, para descartar afección leptomeníngea; además del examen oftalmológico. ${ }^{13,14}$

\section{Diagnóstico diferencial}

El principal diagnóstico diferencial de una malformación capilar en un recién nacido debe establecerse con el hemangioma infantil superficial, que es de crecimiento rápido en los primeros meses de vida a diferencia de las malformaciones capilares que no muestran cambios. ${ }^{2,12}$ 


\section{Tratamiento}

El tratamiento de las malformaciones capilares es mediante el uso de láser de colorante pulsado que causa destrucción de los vasos capilares por calor lumínico, sin embargo, es doloroso y se requieren varias sesiones para obtener una respuesta completa. Puesto que es necesaria la sedación en cada sesión, el tratamiento es poco recomendado en pacientes pediátricos. ${ }^{2,5}$ Algunos autores reportan un requerimiento menor de sesiones si se inicia el tratamiento en etapas más tempranas. ${ }^{12}$ Así mismo, los pacientes con síndrome de Sturge-Weber requieren control de los síntomas neurológicos y oftalmológicos. ${ }^{11,14,15}$

\section{Malformaciones linfáticas}

\section{Epidemiología}

Las malformaciones linfáticas, antes conocidas como "linfangiomas", tienen una incidencia de
1 en 2-4 mil nacidos vivos, sin predilección de género. $^{12}$

\section{Etiología}

Se componen de vasos linfáticos dilatados o lesiones quísticas rodeadas de células endoteliales con fenotipo linfático (CD34+). Son anomalías del desarrollo del sistema linfático que no tienen conexión con el sistema de drenaje. ${ }^{12}$

\section{Manifestaciones clínicas}

Se manifiestan con incremento de volumen subcutáneo, bien delimitado, de consistencia blanda o renitente, generalmente sin cambios en la coloración de la piel. La topografía más frecuente es en el cuello y la región axilar (Figura 3a-b), pero pueden aparecer en cualquier parte del cuerpo. Algunas malformaciones linfáticas tienen en la superficie de la piel vesículas de contenido seroso o hemorrágico, de 1-2 mm de

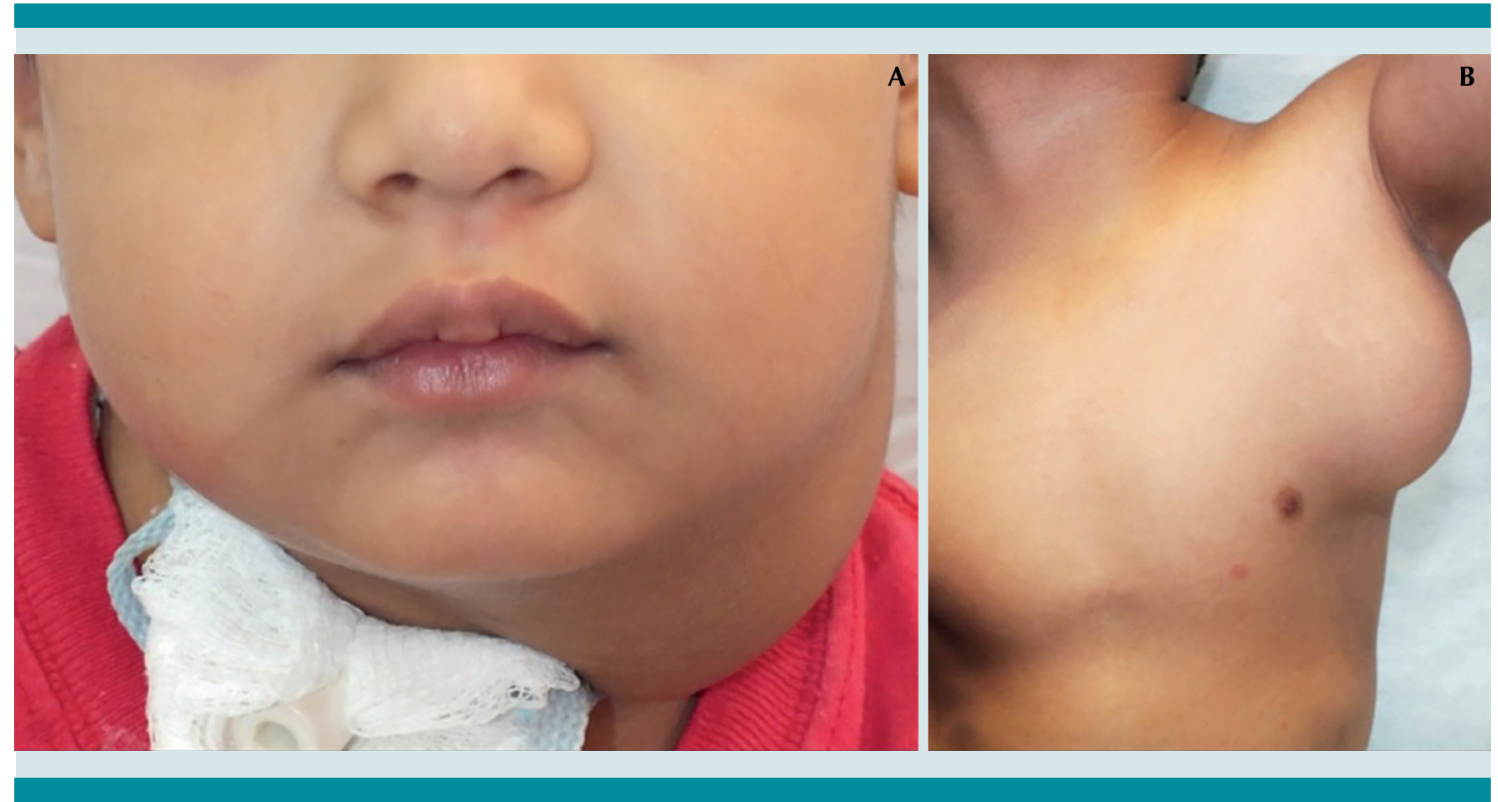

Figura 3. Malformaciones linfáticas. A) en el cuello, B) en la axila. 
diámetro que al romperse condicionan linforrea (salida de líquido linfático). ${ }^{12,22} \mathrm{~A}$ pesar de que por definición están desde el nacimiento, con frecuencia son evidentes después de un traumatismo o proceso infeccioso, por lo que pueden manifestarse en la infancia o adolescencia. La fluctuación del tamaño de la malformación linfática, asociado a procesos infecciosos, es característica. ${ }^{22}$

Dependiendo del tamaño de la cavidad pueden clasificarse en: macroquísticas si son mayores a $2 \mathrm{~cm}$ o microquísticas si miden menos de $2 \mathrm{~cm}$, lo que se define mediante el ultrasonido. ${ }^{12}$

La clasificación detallada de las malformaciones linfáticas se muestra en el Cuadro $3 .^{4}$

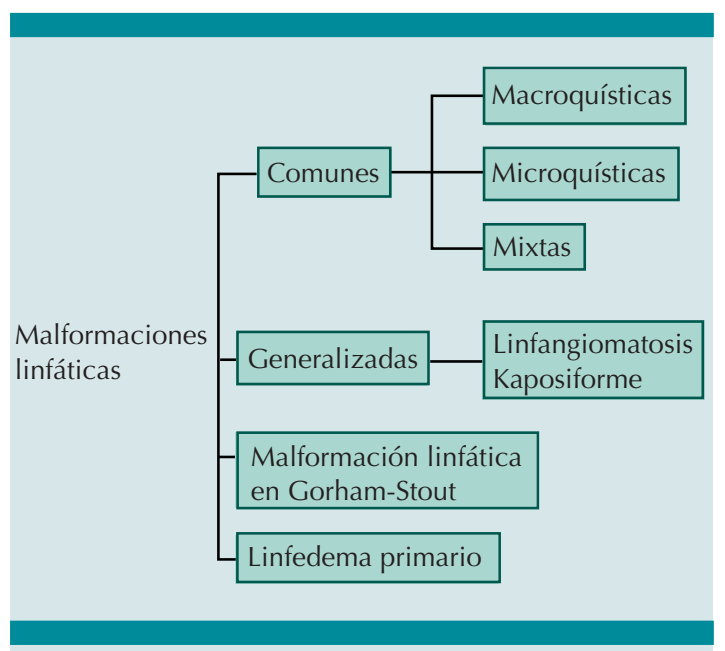

Cuadro 3. Clasificación de malformaciones linfáticas (ISSVA 2018). ${ }^{4}$

\section{Diagnóstico}

El ultrasonido Doppler permite corroborar el diagnóstico, se observan cavidades quísticas, septadas o no, con ausencia de flujo. ${ }^{2,23} \mathrm{La}$ resonancia magnética permite mejor definición de la extensión. ${ }^{24}$

\section{Tratamiento}

Las malformaciones linfáticas pueden tratarse con escleroterapia percutánea con diversos agentes esclerosantes: alcohol absoluto, polidocanol, OK-432 y bleomicina, entre otras, ocasionando daño endotelial directo con obliteración luminal y fibrosis secundarias. ${ }^{25,26}$ La resección quirúrgica en este tipo de malformaciones puede ser difícil ya que no respeta planos anatómicos y tiene una alta tasa de recurrencia. Recientemente se han utilizado los inhibidores de la vía mTOR, sobre todo el sirolimus, para el tratamiento de malformaciones resistentes a manejo convencional con resultados favorables. La dosis es de $0.8 \mathrm{mg} / \mathrm{m}^{2}$ $\mathrm{SC} /$ dosis, 2 veces al día, manteniendo niveles séricos entre $4.5-14 \mathrm{ng} / \mathrm{mL}$ (dependiendo del laboratorio). Los efectos adversos más frecuentes en lactantes y escolares son: estomatitis y diarrea; mientras que en adolescentes puede haber elevación de transaminasas, hipercolesterolemia, neutropenia, y dolor abdominal. ${ }^{27,28}$

\section{Malformaciones venosas}

\section{Epidemiología}

Las malformaciones venosas representan 2/3 partes de todos los casos de malformaciones vasculares, afectan cualquier órgano o tejido y tampoco hay predilección de género. ${ }^{1,5,29}$

\section{Etiología}

Son la consecuencia de un desarrollo vascular aberrante, en donde hay canales venosos dilatados con paredes delgadas y deficientes de músculo liso. ${ }^{12,30}$ El 50\% son esporádicas; sin embargo, los casos familiares tienen herencia autosómica dominante y se asocian con mutaciones en el gen TEK localizado en el cromosoma 9p21 que codifica para el receptor de tirosina cinasa (TIE-2) que se encuentra en la superficie 
de las células endoteliales actuando en conjunto con la angiopoyetina. La mutación de ese gen favorece el incremento en la vascularidad. ${ }^{31}$

La clasificación de las malformaciones venosas más frecuentes incluye (Cuadro 4): ${ }^{4}$

1. Malformación venosa común

2. Malformación venosa familiar mucocutánea

3. Malformación venosa en el síndrome de Bean (Nevo azul ahulado)

4. Malformación glomovenosa

5. Otras malformaciones venosas

\section{Manifestaciones clínicas}

Las malformaciones venosas son congénitas pero pueden no ser evidentes hasta la pubertad o posterior a un traumatismo. Desde el punto de vista clínico se manifiestan con aumento de volumen de aspecto vascular mal delimitado, de consistencia suave y compresible, de color azul-violáceo en la superficie de la piel y, en ocasiones, la visualización de trayectos venosos (Figura 4a). ${ }^{18,32}$ Pueden ser únicas o múltiples, con predominio en la cabeza y el cuello. Estas lesiones incrementan de volumen con maniobras de Valsalva. Cuando afectan la mucosa oral pueden alterar el lenguaje, la dentición o alimentación y las lesiones profundas pueden condicionar deformidad por afectación muscular y ósea. ${ }^{2}$

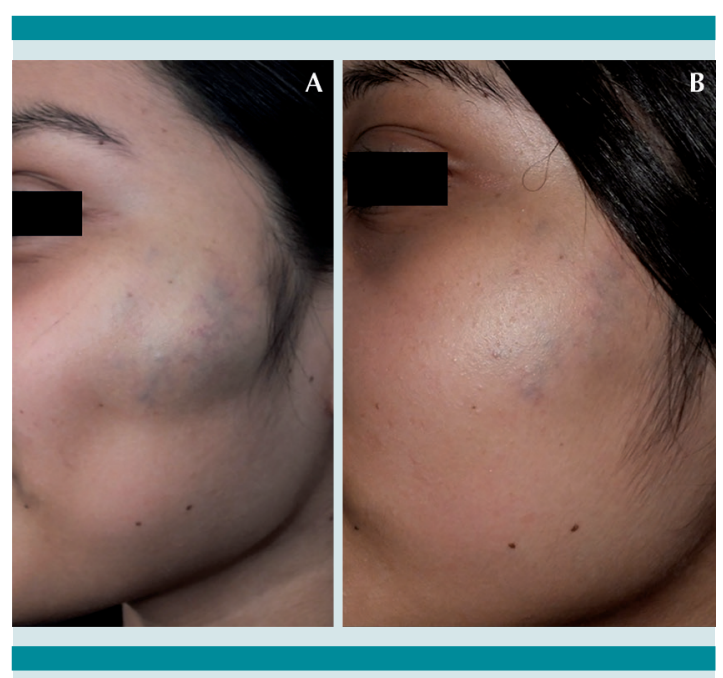

Figura 4. Malformación venosa. A) previa a tratamiento, B) posterior a tratamiento con escleroterapia.

Las malformaciones glomovenosas, también llamadas glomangiomas o angiomas venosos con células glómicas, representan un tipo de malformaciones venosas poco frecuentes (menos de $5 \%$ de los casos). La mayor parte son familiares, de herencia autosómica dominante, secundarias a mutaciones del gen GLMN en el cromosoma 1p21-22 que codifica para la proteína glomulina, que desempeña un papel importante en la diferenciación del músculo liso vascular que da origen a las células glomovenosas. Clínicamente se manifiestan como lesiones nodulares, azul-violáceas, con apariencia "empedrada" más comúnmente en el tronco y las extremidades (Figura 5). Característicamente, son lesiones dolorosas a la palpación. ${ }^{33,34}$ Se han

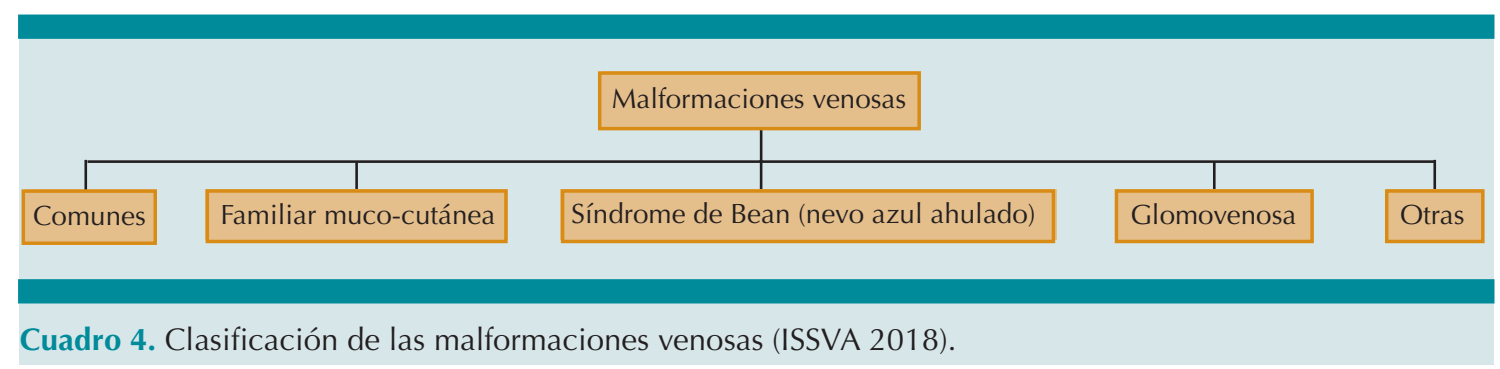


descrito dos subtipos clínicos: la forma solitaria, que aparece en $90 \%$ de los casos y que con frecuencia se localiza en el lecho ungueal y la forma múltiple, que se manifiesta en forma de placa. ${ }^{12,33}$

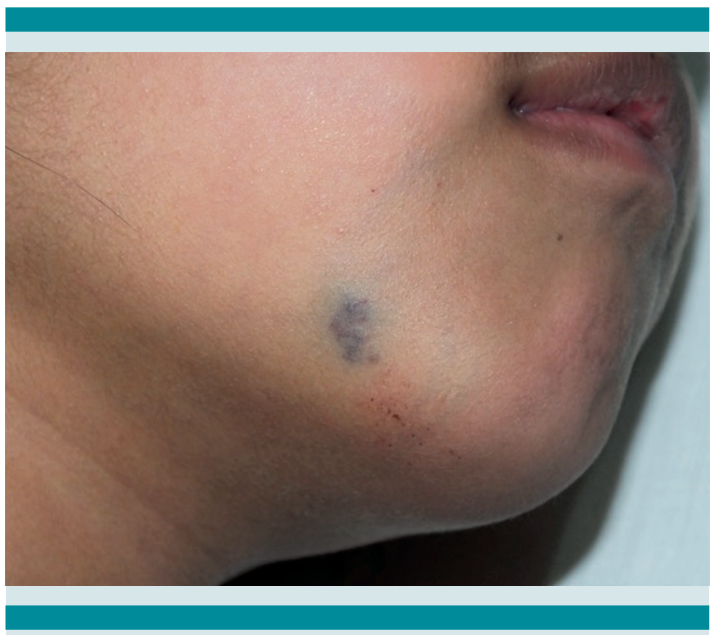

Figura 5. Malformación glomovenosa en la región mandibular derecha.

\section{Complicaciones}

Una complicación frecuente de la malformación venosa es la coagulación intravascular localizada que cursa con disminución de fibrinógeno e incremento de dímero-D, con una cifra normal de plaquetas. Esta coagulación intravascular localizada es secundaria al flujo turbulento y lento dentro de la malformación venosa que predispone a la formación de trombos, también Ilamados flebolitos, los cuales se calcifican en la pared venosa. Estos flebolitos son de consistencia muy firme, palpables y ocasionan dolor y edema. ${ }^{35}$

\section{Diagnóstico}

El ultrasonido Doppler orienta al diagnóstico, se observa una lesión vascular de bajo flujo, característica. La resonancia magnética permite definir la extensión a estructuras profundas. Los flebolitos son evidentes en una radiografía simple. ${ }^{32}$ En el caso de las malformaciones glomovenosas, la histopatología es característica porque se evidencian las dilataciones vasculares rodeadas de células glómicas. ${ }^{34}$

\section{Tratamiento}

El tratamiento es interdisciplinario y depende del tamaño y extensión de la malformación venosa. La escleroterapia se considera el tratamiento de primera elección para las malformaciones venosas sintomáticas (Figura 4b). ${ }^{32,36}$ El uso de medias elásticas de compresión ayuda a disminuir el dolor y el edema. El tratamiento quirúrgico cada vez es menos frecuente porque se asocia con porcentajes elevados de recurrencia, excepto en el caso de las malformaciones glomovenosas solitarias, en las que se considera el tratamiento de elección con el objetivo de controlar el dolor y mejorar los aspectos funcionales. ${ }^{34}$

\section{Malformaciones arteriovenosas}

\section{Epidemiología}

Las malformaciones arteriovenosas son las menos frecuentes, las más complejas y difíciles de tratar. Se presentan por igual en hombres y mujeres; $40-60 \%$ son evidentes al nacimiento y sólo $30 \%$ se manifiestan durante la pubertad.

\section{Etiología}

Las malformaciones arteriovenosas tienen vasos arteriales y venosos anómalos conectados entre sí, sin un lecho capilar intermedio, por una falla en la regresión de los canales arteriovenosos en el plexo embrionario primario. ${ }^{18,37}$ Estas lesiones se caracterizan por una derivación del flujo de alta velocidad y baja resistencia de la vasculatura arterial al sistema venoso, con frecuencia asociadas con lesiones fistulosas. ${ }^{38}$ 


\section{Manifestaciones clínicas}

Desde el punto de vista clínico, las malformaciones arteriovenosas se presentan como neoformaciones subcutáneas mal delimitadas, de coloración azul-violáceo, con trayectos venosos en la superficie, de consistencia firme y a la palpación se detecta un thrill o pulso. La localización más frecuente es en la cabeza seguida del cuello. ${ }^{21,38}$ (Figura 6)

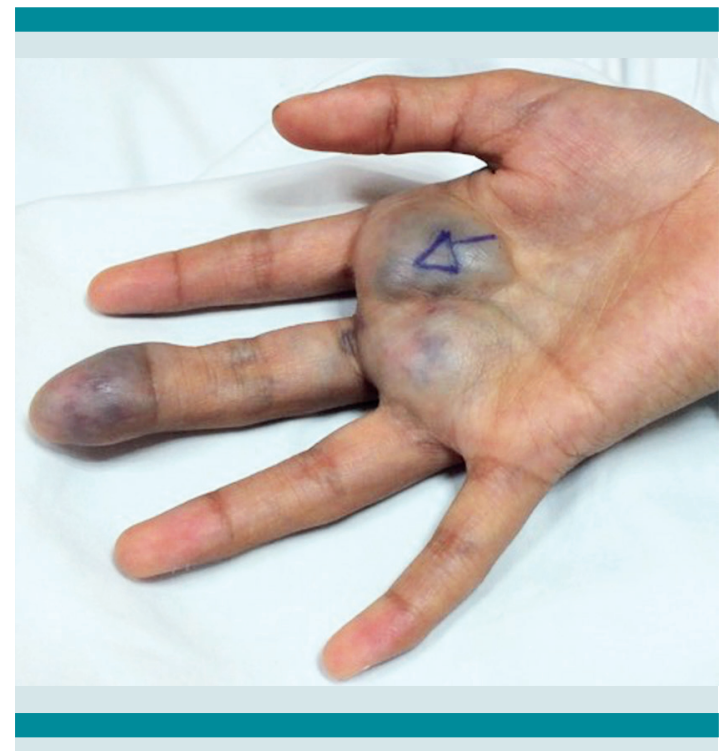

Figura 6. Malformación arteriovenosa en la mano.

Las malformaciones arteriovenosas presentan cuatro estadios evolutivos (fases de Schöbinger): ${ }^{37,38}$

- Estadio 1 = fase quiescente: asintomática.

- Estadio 2 = fase de expansión: crecimiento progresivo de la malformación arteriovenosa que condiciona deformidad e invasión de estructuras profundas, con pulsos.

- Estadio 3 = fase destructiva: destrucción tisular con ulceración, sangrado y necrosis.
- Estadio 4 = fase de descompensación: insuficiencia cardiaca, hipertensión venosa e incluso, la muerte.

\section{Diagnóstico}

En las malformaciones arteriovenosas los estudios de imagen son indispensables para categorizar y planear el tratamiento. El ultrasonido Doppler, a diferencia de las malformaciones linfáticas o venosas, demuestra un alto flujo, la resonancia magnética y la tomografía computada permiten delimitar la extensión y constituyen la única indicación para realizar una angiografía diagnóstica. ${ }^{22,37}$

\section{Tratamiento}

El tratamiento lo realiza el radiólogo intervencionista, con anestesia general, mediante la embolización del vaso o nicho nutricio, colocando diversas sustancias: coils, ónix, cianoacrilato, entre otras, que disminuyen el flujo sanguíneo a otras ramas. En ocasiones se realiza una resección quirúrgica, 1 a 2 semanas después de la embolización, con la que se consiguen mejores resultados y menor sangrado. ${ }^{22,39}$

\section{MALFORMACIONES VASCULARES COMBINADAS}

Las malformaciones vasculares combinadas presentan dos o más lesiones: venocapilares, venolinfáticas, venolinfáticas-capilares, etc.

\section{MALFORMACIONES VASCULARES ASOCIADAS CON OTRAS ANOMALÍAS}

Las malformaciones vasculares mencionadas (simples, combinadas o de grandes vasos) pueden asociarse con otras alteraciones: sobrecrecimiento de tejidos blandos o de hueso subyacente y musculoesqueléticas, entre otras (Figura 7). Los síndromes más frecuentes aso- 


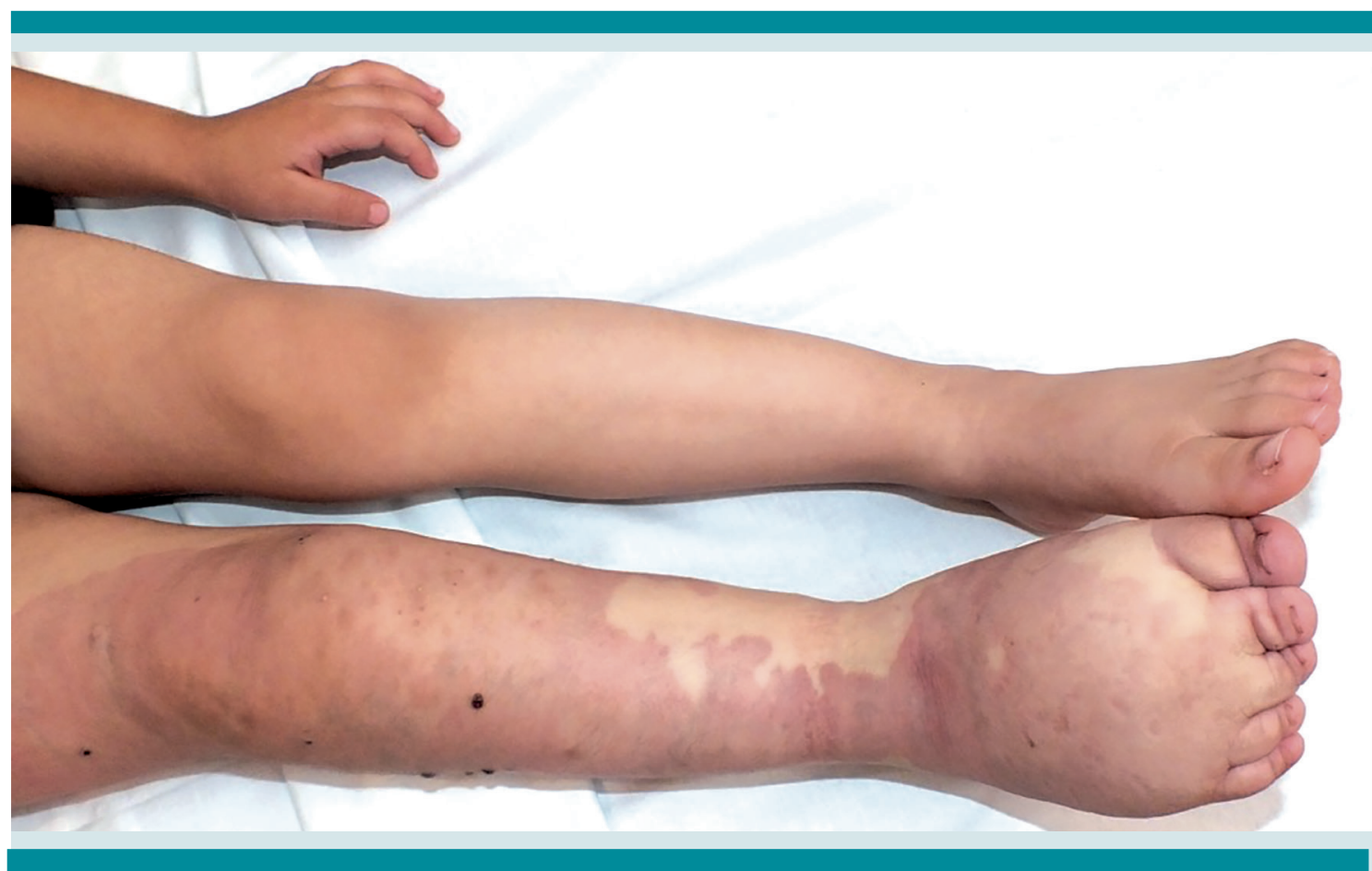

Figura 7. Síndrome de Klippel-Trenaunay.

ciados a malformaciones vasculares se muestran en el Cuadro 5. ${ }^{32}$

A pesar de los avances en estudios de imagen y técnicas de biología molecular, aún existen algunas anomalías vasculares que no logran clasificarse en ninguno de los apartados previos. Por eso, en la clasificación de la ISSVA se encuentra un grupo de anomalías vasculares provisionalmente no clasificables, ya sea porque aún no se logra definir si son tumores o malformaciones o porque su definición y sus características clínicopatológicas no se han dilucidado por completo.

\section{CONCLUSIONES}

La importancia de este segundo artículo de anomalías vasculares radica en poder categorizar, desde el inicio, si se trata de un tumor o una malformación vascular. La clasificación y nomenclatura de las anomalías vasculares, a lo largo del tiempo, ha experimentado grandes cambios, gracias a la genética y al mejor conocimiento de la evolución de cada una de las lesiones vasculares, con el fin de ofrecer un abordaje y tratamiento integral, interdisciplinario y dirigido.

En este artículo se describieron, a detalle, las malformaciones vasculares más frecuentes que componen un amplio y heterogéneo espectro de lesiones que constituyen un desafío diagnóstico y terapéutico para el médico; sin embargo, cada una tiene datos clínicos y evolución característica que hace posible establecer su diagnóstico.

Como se mencionó previamente, todas las malformaciones son defectos del desarrollo vascular por lo que se consideran congénitas, sin em- 
Cuadro 5. Malformaciones vasculares asociadas a otras anomalías (ISSVA 2018)

\begin{tabular}{|c|c|}
\hline Síndrome & Características \\
\hline $\begin{array}{l}\text { Síndrome de } \\
\text { Sturge-Weber }\end{array}$ & $\begin{array}{l}\text { MC en cara + MC leptomeníngea + alteraciones oculares (glaucoma) +/- sobrecreci- } \\
\text { miento de tejidos blandos y/o de hueso }\end{array}$ \\
\hline $\begin{array}{l}\text { Síndrome de Klippel-Trenaunay } \\
\text { (Figura 7) }\end{array}$ & $M C+M V+/-M L+$ sobrecrecimiento de una extremidad \\
\hline $\begin{array}{l}\text { Síndrome de } \\
\text { Parkes-Weber }\end{array}$ & MC + FAV + sobrecrecimiento de una extremidad \\
\hline $\begin{array}{l}\text { Síndrome de } \\
\text { Servelle-Martorell }\end{array}$ & MV en una extremidad + hipotrofia de extremidad \\
\hline $\begin{array}{l}\text { Síndrome de } \\
\text { Maffucci }\end{array}$ & MV + encondromas + hemangioma de células fusiformes \\
\hline $\begin{array}{l}\text { Malformación capilar-macro- } \\
\text { cefalia }\end{array}$ & $M C+$ macrocefalia \\
\hline $\begin{array}{l}\text { Malformación capilar-micro- } \\
\text { cefalia }\end{array}$ & $M C+$ microcefalia \\
\hline Síndrome CLOVES & $\begin{array}{l}M L+M V+M C+/-M A V+\text { sobrecrecimiento lipomatoso + nevo epidérmico + altera- } \\
\text { ciones esqueléticas. }\end{array}$ \\
\hline
\end{tabular}

MC: malformación capilar, MV: malformación venosa, ML: malformación linfática, FAV: fístula arterio-venosa, MAV: malformación arterio-venosa.

bargo, no todas son evidentes al nacimiento. El hecho de que en algunos pacientes las anomalías vasculares se manifiesten en la pubertad, se explica por la existencia de receptores hormonales en los vasos sanguíneos. Una vez que se tiene la sospecha de una malformación vascular es necesario buscar el vaso afectado mediante un estudio de imagen para posteriormente poder realizar una clasificación adecuada y ofrecer un tratamiento específico según el tipo de malformación, tamaño, topografía y asociaciones. En los últimos años, las técnicas de radiología intervencionista ofrecen un amplio abanico de opciones terapéuticas seguras, con el fin de mejorar la calidad de vida de los pacientes. EI tratamiento de los pacientes con anomalías vasculares siempre debe estar a cargo de un grupo médico interdisciplinario.

\section{REFERENCIAS}

1. Wassef M, et al. Vascular anomalies classification: recommendations from the International Society for the Study of Vascular Anomalies. Pediatrics 2015; 136 (1): e20314. https://doi.org/10.1542/peds.2014-3673

2. Foley LS, Kulungowski AM. Vascular anomalies in pediatrics. Adv Pediatr 2015; 62 (1): 227-55. doi: 10.1016/j. yapd.2015.04.009.

3. Enjolras O, Mulliken JB. Vascular tumors and vascular malformations (new issues). Adv Dermatol 1997; 13: 375-423.

4. International Society for the Study of Vascular Anomalies. En: http://www.issva.org.

5. Garzon MC, et al. Vascular malformations: Part I. J Am Acad Dermatol 2007; 56 (3): 353-70. https://doi.org/10.1016/j. jaad.2006.05.066

6. Cordisco RM. Vascular Anomalies in Childhood. CILAD; 2016.

7. Flamme I, et al. Molecular mechanisms of vasculogenesis and embryonic angiogenesis. J Cell Physiol 1997; 173 (2): 206-10.

8. Brouillard P, Vikkula M. Vascular malformations: localized defects in vascular morphogenesis. Clin Genet 2003; 63 (5): 340-51. https://doi.org/10.1034/j.13990004.2003.00092.x

9. Wetzel-Strong SE, et al. The pathobiology of vascular malformations: insights from human and model organism genetics. J Pathol 2017; 241(2): 281-93. https://doi. org/10.1002/path.4844

10. Kanada KN, et al. A prospective study of cutaneous findings in newborns in the United States: correlation with race, ethnicity, and gestational status using updated classifi- 
cation and nomenclature. J Pediatr 2012;161(2):240-5. https://doi.org/10.1016/j.jpeds.2012.02.052

11. Rozas-Muñoz E, et al. Vascular stains: Proposal for a clinical classification to improve diagnosis and management. Pediatr Dermatol 2016; 33 (6): 570-84. https://doi. org/10.1111/pde.12939

12. Del Pozo J, et al. Vascular malformations in childhood. Actas Dermosifiliogr 2012; 103 (8): 661-78.

13. Nguyen $\mathrm{V}$, et al. The pathogenesis of port wine stain and Sturge Weber Syndrome: complex interactions between genetic alterations and aberrant MAPK and PI3K activation. Int J Mol Sci 2019; 20 (9): 2243. doi:10.3390/ijms20092243.

14. Zallmann M, et al. Screening for Sturge-Weber syndrome: A state-of-the-art review. Pediatr Dermatol 2018;35(1):3042. https://doi.org/10.1111/pde.13304

15. Higueros $E$, et al. Sturge-Weber syndrome: A review. Actas Dermosifiliogr 2017; 108 (5): 407-17. https://doi. org/10.1016/j.adengl.2017.03.034

16. Cerisola A, et al. Síndrome de Sturge-Weber sin angioma facial: presentación de un caso clínico con su evolución clínica e imagenológica. Arch Pediatr Urug 2008;79(2):144-9.

17. Juern A, et al. Nevus simplex: A reconsideration of nomenclature, sites of involvement, and disease associations. J Am Acad Dermatol 2010; 63 (5): 805-14. https://doi. org/10.1016/j.jaad.2009.08.066

18. McCuaig C. Update on classification and diagnosis of vascular malformations. Curr Opin Pediatr 2017; 29 (4): 448-54. https://doi.org/10.1097/MOP.0000000000000518

19. Ben-Amitai $D$, et al. Sacral nevus flammeus simplex: the role of imaging. Pediatr Dermatol 2000; 17 (6): 469-71. https://doi.org/10.1046/j.1525-1470.2000.01824.x

20. Del Boz González J, et al. Cutis marmorata telangiectatica congenita. Review of 33 cases. An Pediatr (Barc) 2008;69(6):557-64. https://doi.org/10.1016/S16954033(08)75239-1

21. Basega E, et al. Atlas Clínico de Anomalías Vasculares. 1a ed. Aula Médica, 2016; 692.

22. Radhakrishnan K, Rokcson SG. The clinical spectrum of lymphatic disease. Ann N Y Acad Sci 2008; 1131: 155-84.

23. Mulligan PR, et al. Vascular anomalies: classification, imaging characteristics and implications for interventional radiology treatment approaches. Br J Radiol 2014; 87 (1035): 20130392. https://doi.org/10.1259/bjr.20130392

24. McAlvany JP, et al. Magnetic resonance imaging in the evaluation of lymphangioma circumscriptum. Arch Dermatol 1993;129(2):194-7. doi:10.1001/archderm.1993.0168023007800

25. Acord $\mathrm{M}$, et al Percutaneous treatment of lymphatic malformations. Tech Vasc Interv Radiol 2016; 19 (4): 305-11. https://doi.org/10.1053/j.tvir.2016.10.001
26. Wheeler IS, et al. Ok-432 and lymphatic malformations in children: the starship children's hospital experience. Anz J Surg 2004; 74 (10): 855-8. https://doi.org/10.1111/ j.1445-1433.2004.03115.x

27. Yesil $S$, et al. Single-center experience with sirolimus therapy for vascular malformations. Pediatr Hematol Oncol 2016;33(3):219-25. doi: 10.3109/08880018.2016.1160170.

28. Triana $P$, et al. Sirolimus in the treatment of vascular anomalies. Eur J Pediatr Surg 2017; 27 (1): 86-90.

29. McRae MY, et al. Venous malformations: clinical course and management of vascular birth- mark clinic cases. Australas J Dermatol 2013; 54 (1): 22-30. https://doi. org/10.1111/j.1440-0960.2012.00959.x

30. Gallione $\mathrm{CJ}$, et al. A gene for familial venous malformations maps to chromosome $9 p$ in second large kindred. Med Genet 1995;32(3):197-9.

31. Folkman J, D'Amore PA. Blood vessel formation: what is its molecular basis? Cell. 1996;87(7):1153-5.

32. Garzon MC, et al. Vascular malformations. Part II: associated syndromes. J Am Acad Dermatol 2007; 56 (4): 541-64. https://doi.org/10.1016/j.jaad.2006.05.066

33. Boon LM, et al. Glomuvenous malformation (glomangioma) and venous malformation. Distinct clinicopathologic and genetic entities. Arch Dermatol 2004; 140 (8): 971-6. doi:10.1001/archderm.140.8.971

34. Conde-Taboada A, et al. Multiple, neonatal, self-healing, cutaneous glomuvenous malformations. Indian J Dermatol Venereol Leprol 2017; 83 (2): 226-8. http://www.ijdvl. com/text.asp?2017/83/2/226/196319

35. Zhuo $\mathrm{KY}$, et al. Localised intravascular coagulation complicating venous malformations in children: Associations and therapeutic options. J Paediatr Child Health 2017; 53 (8): 737-41. https://doi.org/10.1111/jpc.13461

36. Gurgacz $S$, et al. Percutaneous sclerotherapy for vascular malformations: A systematic review. Ann Vasc Surg 2014; 28 (5): 1335-49. https://doi.org/10.1016/j. avsg.2014.01.008

37. Lee BB, et al. Consensus document of the International Union of Angiology (IUA)-2013. Current concept on the management of arterio-venous management. Int Angiol 2013; 32 (1): 9-36.

38. Kohout MP, et al. Arteriovenous malformations of the head and neck: natural history and management. Plast Reconstr Surg 1998; 102 (3): 643-54. 10.1097/00006534199809030-00006

39. Han $\mathrm{Y}$, et al. Absolute ethanol embolization combined with surgical resection of scalp arteriovenous malformations: Interim results. J Vasc Interv Radiol 2018; 29 (3): 312-9. https://doi.org/10.1016/j.jvir.2017.10.005 